KRONOS | AGOSTO 2021-ENERO 2022 | vol. $02 \mid$ n. $^{\circ} 1$

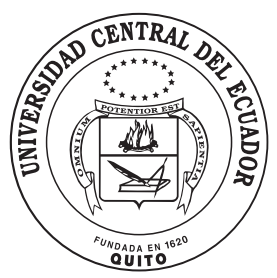

ISSN-I 2631-2840 | ISSN-E 2631-2859

VIRTUAL CLASSES AND THEIR EFFECT ON THE PHYSICAL AND MENTAL STATE OF UNIVERSITY STUDENTS

\title{
CLASES VIRTUALES Y SU EFECTO EN EL ESTADO FISICO Y MENTAL DE LOS ESTUDIANTES UNIVERSITARIOS
}

Paul Arias

Universidad de las Fuerzas Armadas, ESPE (Ecuador)

https://orcid.org/0000-0002-1653-458X

Anahí Armas

Universidad de las Fuerzas Armadas, ESPE (Ecuador)

https://orcid.org/0000-0001-9540-0119

\author{
Alexis Arévalo \\ Universidad de las Fuerzas Armadas, ESPE (Ecuador) \\ https://orcid.org/0000-0001-7399-4489
}

Recibido: 28 de marzo 2021

Aprobado: 26 de julio 2021

DOI: 10.29166/kronos.v2i1.3026

\section{RESUMEN}

El presente artículo tiene como objeto analizar el estado físico y mental de los estudiantes de primero y segundo semestre de Comercio exterior y de Ingeniería Mecánica de la Universidad de la Fuerzas Armadas espe durante la pandemia. Para lo cual se realizó una encuesta en línea mediante un análisis deductivo con enfoque cuantitativo. La investigación fue realizada durante los meses de noviembre 2020 a febrero 2021, dando como resultado que la mala postura y la poca actividad física de los estudiantes durante y después de sus clases virtuales, ha causado diversos problemas físicos tales como fatiga ocular, dolores musculares, estomacales y de cabeza. Además de trastornos mentales como depresión, insomnio y ansiedad.

\begin{abstract}
The purpose of this article is to analyze the physical and mental state of the first and second semester students of Foreign Trading and of Mechanical Engineering of the Universidad de las Fuerza Armadas espe during the pandemic. For which an online survey was conducted using a deductive analysis with a quantitative approach. The research was carried out during the months of November 2020 to February 2021, resulting in the students' poor posture and little physical activity during and after their virtual classes has caused various physical problems such as eye strain, sore muscle, stomachache, and headache. Additionally, mental disorders such as depression, insomnia, and anxiety are observed.
\end{abstract}

palabras Clave Pandemia, clases virtuales, estudiantes, estado físico y mental.

KEYWORDS Pandemic, virtual classes, students, physical and mental state. 


\section{INTRODUCCIÓN}

Hoy en día existe una clara evidencia de que llevar una vida activa mejora la salud, el bienestar y la calidad de vida a cualquier edad, permitiendo vivir más tiempo de manera independiente y ayudando a prevenir enfermedades crónicas. Incorporar a la vida cotidiana algunas actividades cotidianas como caminar, subir escaleras, correr, jugar, bailar, montar en bicicleta, nadar, etc., mejora la salud física y mental de gente joven y adulta. En el transcurso de la pandemia los niveles de actividad física en los estudiantes disminuyeron; afectando negativamente el estado de ánimo y el bienestar. En base a esto, se analizaron los problemas de salud más comunes generados durante la educación virtual. Se realizó una encuesta en línea a un grupo de estudiantes de la Universidad de las Fuerzas Armadas ESPE en el primer y segundo semestre de Comercio Exterior y de Ingeniería Mecánica, mediante un análisis deductivo con un enfoque cuantitativo, el cual ayudó a obtener los datos necesarios para desarrollar el proceso de recolección y estudio de datos. La investigación fue realizada durante los meses de noviembre 2020 a febrero 2021 y se centró en los problemas físicos y mentales que tiene la mayoría de los estudiantes encuestados, mismos que eran problemas preexistentes, pero en menos cantidad.

\section{MARCO TEÓRICO}

\section{Defectos refractivos en los estudiantes al perman- ecer largos periodos de tiempo frente a dispositivos electrónicos}

Debido a la inesperada situación mundial, la población se vio obligada a acoplarse a las nuevas tecnologías y metodologías en el ámbito de la educación en todos sus niveles y el Ecuador no fue la excepción. En el país, maestros y estudiantes se vieron en la necesidad de aprender y emplear nuevos métodos de enseñanza y aprendizaje, siendo parte de ello la implementación total de la tecnología. La cual, a pesar los prometedores beneficios, también trae algunas desventajas. Los defectos visuales o defectos refractivos debido al excesivo desgaste visual son una de las desventajas que acarrea la exposición continua de las pantallas. La Organización Mundial de la Salud calcula que a nivel mundial más de 314 millones de personas viven con una deficiencia visual severa. De ellos, 37 millones están ciegas y 124 millones padecen baja visión. Aproximadamente, 153 millones tienen alguna pérdida de visión debido a los defectos de refracción, problemas solucionables con un examen ocular y gafas. El $99 \%$ de los ciegos viven en los países en desarrollo. Cuatro de cada cinco de ellos pierden la vista innecesariamente (Molina Curbelo, 2017). Con frecuencia, los defectos de refracción más comunes en los estudiantes son la miopía, el astigmatismo y la hipermetropía, en algunos países de América Latina se considera a la hipermetropía, y a la miopía como la segunda causa de la ceguera (Roselló Leyva, 2011).

\section{MIOPÍA}

La miopía es una alteración refractiva la cual viene dada por un exceso longitudinal ocular o por una desmedida curvatura del cristalino, haciendo que el dioptrio tenga una variación en su aumento (Ramírez, 2018). Un ojo miope es aquél que forma las imágenes procedentes del infinito - desde unos $5 \mathrm{~m}$, igual que en las cámaras de fotos - antes de la retina. Por lo tanto, la imagen que llega a la retina es borrosa, transmitiendo de esa manera al cerebro. Las personas con miopía presentan dificultad para enfocar bien los objetos lejanos lo que significa un déficit de agudeza visual (Arancón, 2014).

Existen dos tipos de miopía: la miopía simple que está entre las 5 y 6 dioptrías y evoluciona hasta las 22 a 24 años; la miopía patológica (magma o progresiva) está por el contrario sobrepasa las 6 dioptrías y se cree que está causada por una alteración en el segmento posterior del ojo (Arancón, 2014). Una de sus causas puede ser hereditaria, pero otra muy importante podría ser el hecho de la realización de tareas que impliquen una visión cercana durante largos periodos de tiempo frente a objetos que puedan afectar a la visión (Arancón, 2014).

\section{ASTIGMATISMO}

Condición que causa una visión borrosa debido a la forma irregular de la córnea, la cubierta transparente del ojo, y una córnea de forma irregular impide que la luz se enfoque correctamente en la retina, que es la superficie sensible a la luz en la parte posterior del ojo y como consecuencia la visión se vuelve borrosa sin importar la distancia (Álvarez y Torres, 2015).

Existen dos tipos de astigmatismo: astigmatismo puro, uno de los meridianos se enfoca sobre la retina y el otro según la curvatura puede incidir delante de la retina (astigmatismo miópico) y detrás de la retina (astigmatismo hipermetrópico) (Arancón, 2014); y astigmatismo mixto: un eje se enfoca delante de la retina y el otro detrás.

Las causas del astigmatismo, según Arancón (2014), son las siguientes: 1 . astigmatismo regular, suele ser congénito y no crónico, se debe a que se forman dos ejes en el ojo que refractan la luz con distinta intensidad, debido a que la curvatura de la córnea está muy modificada; según se refracta la luz en los meridianos podemos distinguir los siguientes tipos de astigmatismo regular: astigmatismo frecuente (la luz se refracta con mayor intensidad en el meridiano vertical); astigma- 
tismo poco frecuente (se refracta más la luz en el eje horizontal); 2. astigmatismo irregular: se debe a que se ha modificado la curvatura de la córnea por lesiones, intervenciones quirúrgicas, etc.; en este caso se forman dos ejes irregulares que refractan la luz con una intensidad y dirección muy variada; y 3. astigmatismo temporal: debido a una intervención quirúrgica se puede ver retraída la capa en la córnea, lo que provoca modificaciones en la refracción de la luz que suelen ser temporales y arreglarse de manera espontánea.

\section{HIPERMETROPÍA}

La hipermetropía está conformada por rayos de luz semejantes los cuales son emitidos por un objeto en el infinito, dichos rayos se posicionan por detrás de la retina, generando las siguientes consecuencias. $\mathrm{Al}$ focalizar los objetos que se encuentran a distancias próximas llegan borrosos al ojo, pero los objetos que se encuentran a distancias lejanas son focalizados nítidamente por el globo ocular (Ramírez Moyano, 2018). Existen dos factores principales de riesgo para la hipermetropía, el primero es si sobrepasas la edad de 40 años y el segundo es el factor hereditario quiere decir que si un miembro de la familia lo tiene podría transmitirlo (Arancón, 2014).

\section{Problemas de salud ocasionados por movimientos repetitivos}

Estar sentado durante periodos de tiempo prolongados implica un mayor riesgo para eventos cardiovasculares (hasta un $125 \%$ mayor riesgo) y hasta de 50\% más de fallecer por cualquier causa (alteraciones metabólicas y neoplasias) (Kimmorley, 2014). La actividad física y el ejercicio no ayudan a compensar del todo el riesgo cardiovascular al estar sentado durante periodos prolongados, en otras palabras, las personas activas físicamente presentan en menor medida los efectos adversos de estar sentados durante períodos prolongados en comparación con las personas sedentarias (Kimmorley, 2014).

\section{INACTIVIDAD MUSCULAR}

Con relación a la inactividad muscular, los factores adicionales que promueven trastornos musculoesqueléticos. Los músculos deben estar activos para seguir funcionando, al igual que los tendones y los huesos. No activarlos da como resultado la pérdida de la forma física y defectos estructurales y funcionales. En estas condiciones, los músculos no pueden estabilizar adecuadamente las articulaciones y los ligamentos (Luttmann, Jager y Griefhan, 2004).

\section{MOVIMIENTOS REPETITIVOS}

El ejercicio repetitivo prolongado, ya sea que se carguen objetos o no, puede causar trastornos musculoesqueléticos. Se habla de tareas repetitivas cuando la misma parte del cuerpo se mueve una y otra vez sin poder al menos descansar un rato o cambiar de movimiento (Luttmann, Jager y Griefhan, 2004).

\section{POSIBLES CONSECUENCIAS}

Enfermedad venosa crónica. Una de las principales causas de esta enfermedad es estar parado o sentado por mucho tiempo sin caminar, ya que se disminuye el retorno venoso y se aumenta la presión en las venas de las extremidades inferiores, acumulando la sangre. Los músculos en las piernas juegan un papel muy importante en la circulación, ya que al estar en movimiento actúan como bomba para mover la sangre de las piernas de vuelta al corazón (Elorza, n. d.).

Alteraciones en el metabolismo. La inactividad física puede inducir efectos negativos en los procesos celulares de acción relativamente rápida en los músculos esqueléticos u otros tejidos que regulan los factores de riesgo como los triglicéridos plasmáticos y el colesterol HDL y disminución en la respuesta a la insulina, todo esto hace que el metabolismo se vuelva más lento llevando a consecuencias como el aumento de peso, problemas en el sistema digestivo hasta problemas cardiovasculares y desarrollo de enfermedades metabólicas como diabetes mellitus o síndrome metabólico (Hamilton, 2007).

Alteración en la postura. Molestias agudas asociadas a fatiga muscular que se reflejan en incomodidad, tensión muscular y dolor, especialmente en el cuello y la cintura. Al estar sentadas, las personas tienden a relajarse (se hunden en el asiento) y sus músculos dejan de realizar el trabajo de mantener la postura. La mayor parte del peso se pone directamente en la columna y la pelvis, de modo que la curvatura normal en «S» de la columna se convierte en una «C», que no es una postura suficientemente fuerte para soportar la presión sobre los cuerpos y discos intervertebrales (Zapata, 2014).

Efectos musculoesqueléticos. Cuando no se evita la inactividad prolongada, se produce una pérdida de la forma y la función, donde el músculo no puede estabilizar las articulaciones ni los ligamentos produciendo: dolor, limitación de los arcos de movimiento, inestabilidad de las articulaciones y esfuerzo excesivo. Las alteraciones músculo esqueléticas son muy dolorosas, comúnmente incapacitantes y generalmente tienen un comienzo gradual como el síndrome del túnel carpiano, tendinitis, ciática, hernias de disco, y el dolor de espalda baja, éstas se producen cuando las 
capacidades físicas del trabajador no son compatibles a los requisitos físicos del trabajo (Elorza, n. d.).

Efectos psicológicos. Estar demasiado tiempo sentado no solo afecta la salud física, sino que también afecta la salud mental. Se ha demostrado la gran incidencia que tiene permanecer sentado en enfermedades como depresión, insomnio y ansiedad, lo que ocasiona que estas personas no desean levantarse en las mañanas para ir a trabajar, estudiar o realizar sus labores diarias; a diferencia de las personas que tienen una adecuada actividad física en quienes incluso si ya tienen depresión, el ejercicio podría aliviar sus síntomas o prevenir síntomas futuros. Estar sentados frente a un computador por largos periodos de tiempo afecta la comunicación que tienen las personas con los demás, ya que en lugar de conectarse con el entorno, el individuo se concentra por mucho tiempo en una misma tarea dejando a un lado lo que lo rodea, quitando así la posibilidad de crear conexiones interpersonales. Además de estos trastornos las personas que día a día tienen que enfrentar una alta carga laboral pueden llegar a experimentar el síndrome de Burnout, éste se refiere al estrés laboral crónico, que lleva al cansancio emocional, despersonalización y menor realización personal. Se caracteriza por un agotamiento progresivo tanto físico y mental, falta de motivación por las tareas que se realizan y principalmente cambios en el comportamiento o actitud de la persona, por ejemplo, cambios en el estado de ánimo, donde la persona está más irritable y de mal humor, muestran indiferencia por lo que hacen; desmotivación, estas personas pierden cualquier tipo de interés por trabajar, cumplir sus metas y objetivos; agotamiento mental, haciendo que cada vez tengan menos resistencia a las situaciones de estrés o de mayor exigencia laboral y, por último, falta de energía, menor rendimiento y deterioro cognitivo, causando disminución en la concentración, memoria y la capacidad para desarrollar diferentes actividades (Elorza, n. d.).

\section{Ejercicios para combatir el sedentarismo}

EJERCICIOS DE CALENTAMIENTO Y ESTIRAMIENTO PARA MEJORAR LA ERGONOMÍA

El ejercicio regular, como caminar, nadar o ir en bicicleta, ayudará al cuerpo a mantenerse sano, mientras que los ejercicios de estiramiento y tonificación ayudarán a acondicionar los músculos, prevenir lesiones y mantener una buena postura.

Meditación. Es una práctica en la que los individuos entrenan su pensamiento o inducen la conciencia para lograr ciertos beneficios específicos, como reconocer el contenido psicológicamente o finalmente lograr el propósito sin reconocer el contenido de manera inconsciente. El término meditación se refiere a una amplia gama de prácticas, incluidas técnicas diseñadas para promover la relajación, desarrollar energía interior o fuerza vital (Qì, ki, chi, prā̄a, yoga, etc.) y desarrollar compasión, amor, paciencia, generosidad y perdón. La meditación tiene como objetivo permitir que las personas se concentren sin esfuerzo, enfocándose en mantener un estado de felicidad en cualquier actividad de la vida.

Entrenamiento cardio hit. Básicamente, la idea del entrenamiento a intervalos de alta intensidad o HIIT implica combinar períodos cortos de entrenamiento cardiovascular muy intenso (alrededor del $80-90 \%$ de nuestra frecuencia cardíaca) con otros períodos cortos de intensidad media o baja (50-60\%). Según investigaciones, este tipo de entrenamiento cardiovascular, puede activar la producción de mitocondrias, que son los orgánulos encargados de aportar la mayor parte de la energía necesaria para la actividad celular, puede mejorar la utilización de la glucosa. Durante varias semanas puede mejorar la capacidad de oxidar la grasa (utilizar el exceso de grasa en el cuerpo como energía) y aumentar el valor máximo de vo2 (mejorar la salud cardiovascular) resistencia aeróbica.

\section{METODOLOGÍA}

Debido a que la pandemia mundial (covid -19), no hay muchos datos estadísticos en el Ecuador. Para la investigación, se realizó una encuesta en línea a un grupo de estudiantes de la Universidad de las Fuerzas Armadas ESPE en el primer y segundo semestre de comercio exterior y mecánica, mediante un análisis deductivo con un enfoque cuantitativo. La investigación fue realizada durante los meses de noviembre 2020 a febrero 2021. La población universal total de las carreras de Comercio Exterior e Ingeniería Mecánica de primer y segundo semestre es de: $1{ }^{\text {er }}$ curso comercio: 30 estudiantes, $2 .^{\circ}$ curso comercio: 38 estudiantes, $3 .^{\text {er }}$ curso Mecánica: 40 estudiantes, y

4. ${ }^{\circ}$ curso Mecánica: 39 estudiantes.

Teniendo un total de población: 147 estudiantes el cual respondieron 102 estudiantes de la población seleccionada previamente. Siendo así se logró obtener los siguientes resultados, en las preguntas de la encuesta realizada.

\section{ANÁLISIS DE DATOS}

Para el análisis de los datos se tomó en consideración las respuestas de la encuesta realizada a los estudiantes de la ESPE. De la cual se obtuvieron algunos datos relevantes. Con relación al tiempo que los estudiantes permanecen a un dispositivo electrónico durante sus clases y después de ellas, los estudiantes mencionan que el $83,3 \%$ ( 85 personas de 102) pasa más de 8 horas en adelante en la misma posición y un $12,7 \%$ trabaja 
frente a un dispositivo electrónico (ver Figura 1). Esto significa que es más de la mitad de la población la que pasa más del tiempo en la misma posición. Se debe saber que «las investigaciones han relacionado el hecho de estar sentado durante tiempos prolongados con una serie de problemas de salud» (Laskowski, 2020).

Dentro de las afecciones más comunes por la exposición prolongada a los dispositivos electrónicos y posición sedentaria por largos periodos de tiempo a causa de la modalidad de educación virtual, entre los estudiantes que encontró que las afecciones más recurrentes fueron la fatiga ocular y el dolor de espalda cada una con un $75,5 \%$. El $66,7 \%$ de los estudiantes también mencionaron que tuvieron constantes dolores de cabeza. La última de las opciones de respuesta propuestas, y aunque no parezca tener mucho sentido es el dolor estomacal, pero después de un análisis este síntoma puede ser producido por alimentarse en horarios no recomendados o simplemente omitir comidas por falta de tiempo, aclarado esto, los resultados obtenidos fueron del 14,7\% (ver Figura 2).

Debido a que la fatiga ocular muchas veces desencadena el dolor de cabeza y es importante conocer la magnitud de éste. Teniendo como opciones de respuesta una escala del 1 al 5, considerando así el 1 como el dolor más bajo y el 5 como intenso, tuvimos los siguientes resultados: suave-leve-moderado-severo-intenso. Con respecto a la percepción del dolor en la categoría 1 suave tenemos un resultado del 9,3\%. Con una percepción leve del dolor se aprecia un 16,3\% de los encuestados. El porcentaje es mayor al llegar a la escala de moderado con un $55,8 \%$ de respuesta a este nivel; quienes previamente debieron marcar dolor de cabeza o fatiga ocular en la segunda pregunta de la encuesta aplicada. A continuación el 12,8\% refiere molestia severa y apenas 5 personas de 86 mencionan sufrir de dolores de cabeza en escala de intenso (ver Figura 3).

Pese a que en la encuesta el 100\% de los estudiantes están de acuerdo en que el ejercicio es una base fundamental para prevenir los síntomas de estrés, los datos arrojan un porcentaje negativo, ya que un $24,5 \%$ realiza ejercicios de meditación 2 veces a la semana, el
$6,6 \%$ lo hace 3 veces a la semana, el $4 \%$ lo hace 5 veces a la semana que es una cantidad baja. Finalmente, la entrevista demostró que un $64,7 \%$ de estudiantes nunca realiza ejercicios de meditación (ver Figura 4).

Otro de los factores en el análisis propuesto se basa en el estado mental de los individuos por la exposición prolongada a una misma postura como lo menciona Elorza (n. d.). Los estudiantes universitarios han vivido esta realidad desencadenando en ellos diferentes trastornos como los expuestos en esta pregunta. Por cada 102 estudiantes de primero y segundo semestre de la carrera de Ingeniería Mecánica y Comercio Exterior, 82 estudiantes padecen de algún trastorno mental debido a las condiciones actuales de estudio. De este número de estudiantes aproximadamente la tercera parte $(32,9 \%)$ de la población encuestada padece depresión. En el caso del insomnio, la encuesta nos muestra que el $53,7 \%$ sufre de este trastorno. También $57,3 \%$ de los estudiantes sufren de ansiedad. Finalmente, el 12,25\% sufre de algún trastorno de comunicación debido al confinamiento (ver Figura 5).

\section{CONCLUSIONES}

Finalizado el análisis, se puede concluir que los estudiantes de los primeros niveles la Universidad de las Fuerzas Armadas ESPE, al estar expuestos a movimientos repetitivos por periodos de tiempo prolongados, han desarrollado afecciones físicas como fatiga ocular, dolores de cabeza, dolores de espalda, dolores estomacales, etc. Estos tres primeros factores tienen una incidencia mayor al 60\% lo que corrobora la información obtenida de las encuestas.

Adicionalmente, el 80,39\% de los estudiantes encuestados padece un trastorno psicológico, entre los principales se encuentran el insomnio, depresión y ansiedad. Finalmente, la poca o nula actividad física y de meditación ahonda más estas problemáticas. Para mejorar estos aspectos se aconseja a la comunidad universitaria realizar pausas activas o si es posible practicar algún tipo de meditación durante y después de sus jornadas académicas. 
1. ¿Cuántas horas permanece estudiando frente a un dispositivo electrónico? 102 respuestas

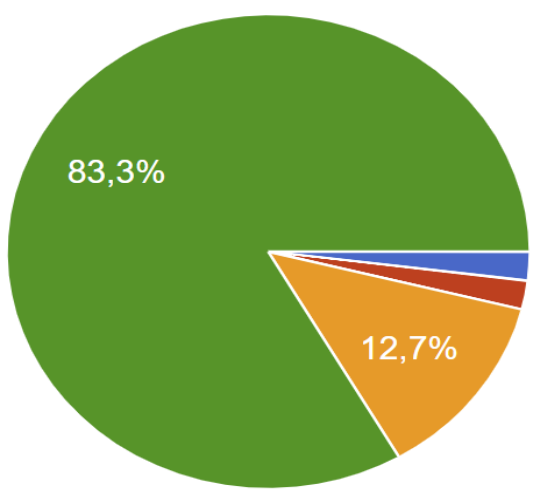
4 horas o menos
6 horas
8 horas o más

5 horas

Figura 1. Tiempo frente a un dispositivo electrónico.

2. Ha percibido algún síntoma como los siguientes: 102 respuestas

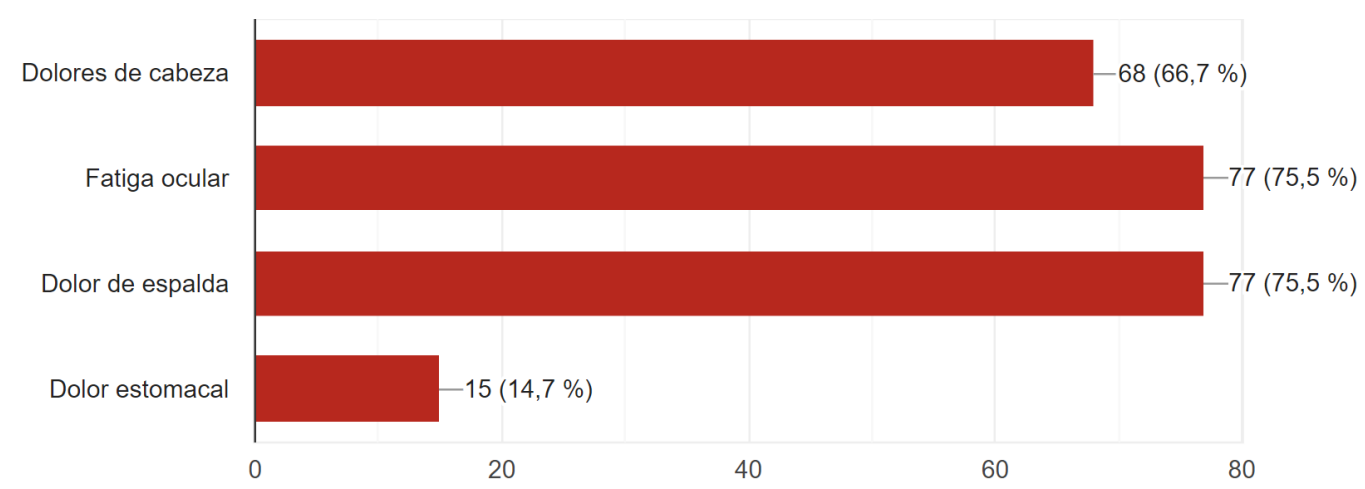

Figura 2. Síntomas

3. Califique su dolor de cabeza (si es que lo siente): 86 respuestas

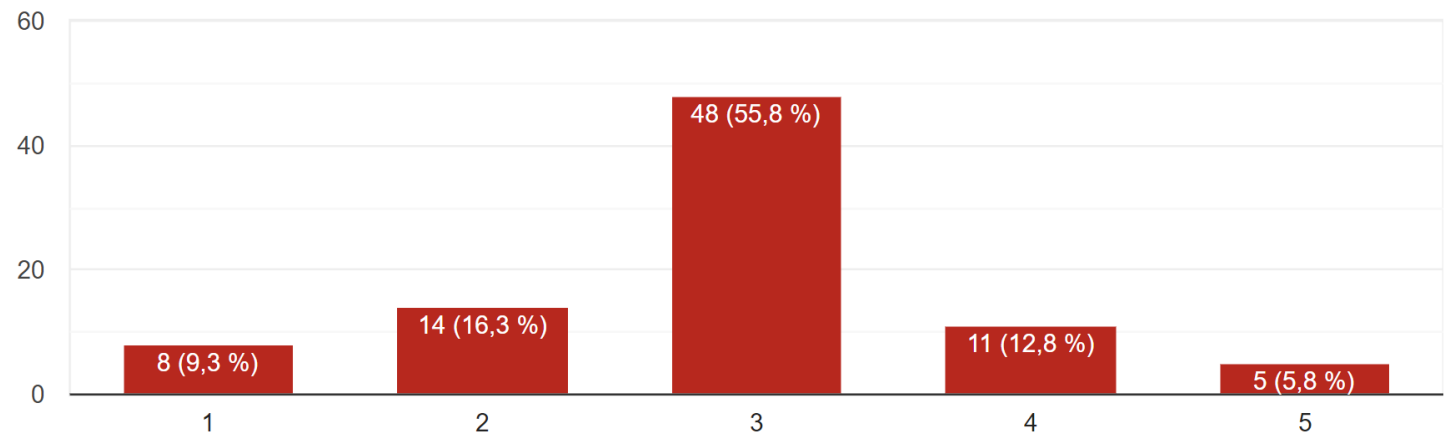

Figura 3. Intensidad 
5. ¿Con qué frecuencia usted realiza ejercicios de meditación a la semana? 102 respuestas

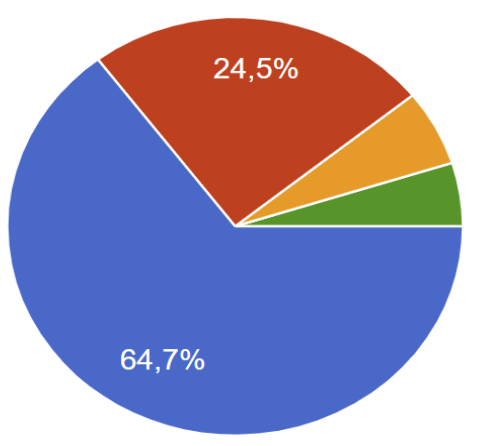

Nunca

2 veces

3 veces

5 veces

Figura 4. Frecuencia

7. Marque las patologías que cree usted que padece a nivel psicológico. (si las padece). 82 respuestas

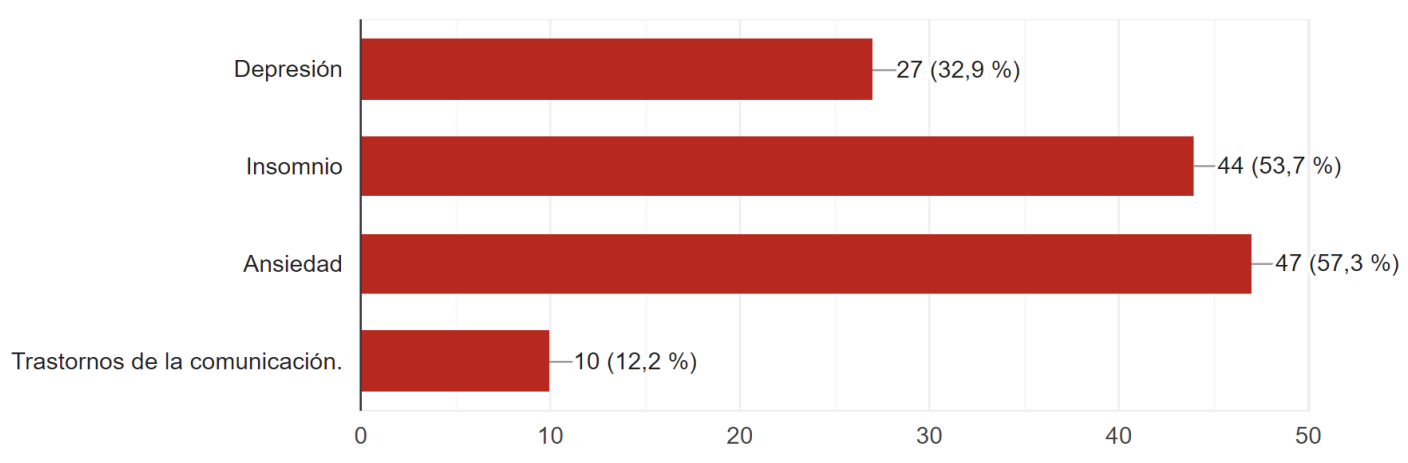

Figura 5. Patologías 


\section{REFERENCIAS}

Álvarez Cárdenas, N. D. R. y Torres Ríos, C. S. (2015). Simulación y modelamiento del ojo humano como herramienta para la prevención del glaucoma a través la medición de la presión intraocular. Eduardo Pinos Vélez. https://dspace.ups.edu.ec/bitstream/123456789/8963/1/ UPS-CT005239.pdf

Arancón, C. (2014, diciembre 19). El ojo humano y sus defectos. https://www.ucm.es/data/cont/docs/136-2015-0129-el\%20ojo\%20humano\%20y\%20sus\%20defectos.pdf Elorza, N., Ortiz, M., Díaz, J., Gonzales, M., Rendón, E. y Rodríguez, M. (2017). Sedestación o permanecer sentado mucho tiempo: riesgo ergonómico para los trabajadores expuestos. CEs Salud Pública, 8(1), 134-147. https:// dialnet.unirioja.es/servlet/articulo? codigo $=6176889$

Hamilton, M., Hamilton, D. y Zderic, T. (2007, noviembre). Papel del bajo gasto energético y estar sentado en la obesidad, el síndrome metabólico, la diabetes tipo 2 y las enfermedades cardiovasculares. American Diabetes Association. https://diabetes.diabetesjournals.org/content/56/11/2655.long\#ref-55

Kimmorley, S. (2014, marzo 17). 14 cosas horribles que pueden suceder si te sientas en tu escritorio durante demasiado tiempo. Business Insider Australia. https://www. businessinsider.com/14-horrible-things-that-can-happe n-if-you-sit-at-your-desk-for-too-long-2014-3?fbclid=IwAR3aKgKLNMOQR4yR3jP4mwuIgc9QMEvfiufN2cffZZ_J6Q5pRCVqpOvvfm8

Luttmann, A., Jager, M. y Griefhan, B. (2004). Prevención de trastornos musculoesqueléticos en el lugar de trabajo (5. ${ }^{\mathrm{a}}$ ed.). Organización Mundial de la Salud. https://www. who.int/occupational_health/publications/en/pwh5sp.pdf Macancela Sarango, L. E. (Junio 3, 2020). https://www.veris. com.ec/nutricion-tiempos-covid-19/

Molina Curbelo, D. (2017). Comportamiento de los defectos refractivos en estudiantes de la escuela primaria Ignacio Agramonte y Loynaz. MediSur, 15(2), 8. http://scielo. sld.cu/scielo.php?pid=S1727-897X2017000200009\&sc ript $=$ sci_arttext\&tlng $=\mathrm{pt}$

Ramírez Moyano, V. A. (2018). Defectos refractivos (miopía, hipermetropía y astigmatismo) desde la óptica geométrica, una experiencia de aprendizaje activo con un grupo de estudiantes del tópico de óptica física de la Universidad Pedagógica Nacional. (s. d.).

Roselló Leyva, D. A. (2011). Defectos refractivos más frecuentes que causan baja visión. Revista Cubana de Oftalmología, 24(2), 8. http://scielo.sld.cu/scielo.php?pid=S0 864-21762011000200007\&script=sci_arttext\&tlng=pt

Torres, K., Dias, E. A. e Iparraguirre, J. P. (2008). Riesgos ergonómicos físicos que influyen en trastornos músculo esquelético de enfermeros que laboran en centro quirúrgico del hospital Cayetano Heredia. 1(1), 35. http:// repositorio.upch.edu.pe/bitstream/handle/upch/3619/ Riesgos_CepidaTorres_Kely.pdf?sequence=1\&isAllowed=y

Zapata, G. (2014). Guía de Orientación (vol. 1). Instituto de Mayores y Servicios Sociales (IMSERSO). https://fiapam. org/wp-content/uploads/2015/07/600093_postural_demencia.pdf 